\title{
Hydrodynamics of DARPA SUBOFF Submarine at Shallowly Immersion Conditions
}

\author{
D.V. Efremov \& E.M. Milanov \\ Bulgarian Ship Hydrodynamics Centre, Varna, Bulgaria
}

\begin{abstract}
Recently, the submarine missions are often evolving into operating to littoral areas, which require operating in shallow water. Such shallow water operations strongly contrast with the traditional ones due to the effect of a close to free water surface expressed mainly by surface suction force. This influence is particularly important for submarine maneuverability accounting for restricted area available. The prediction of submarine behavior in similar conditions requires adequate mathematical model and understanding of the additional hydrodynamic load generated near the surface region.

The paper is aimed for better understanding of these issues and relating to development of a submarine simulation model, the experimental program of towing and PMM captive tests of DARPA Suboff submarine model were conducted at a towing tank. The influence of phenomenon such as effect of a close to free surface and Froude number at hydrodynamic forces and moments including control surfaces effectiveness were investigated and also was estimated directional stability of motion in horizontal plane.
\end{abstract}

\section{INTRODUCTION}

The interest in hydrodynamics of floating, fully submerged bodies is increasing due to the development of floating facilities for research and extraction of marine resources, maintenance of underwater pipelines, production platforms and so on. That revives interest of researchers on one fundamental problem of floating submerged bodies the effect of free surface depending on body immersion. This problem and actuality arise from the fact that usually underwater bodies and appliances are tested mainly for deep water, where the resistance and all other forces and moments have a viscous nature, i.e. wave-making and induced wave forces are ignored. Such bodies, however the shallow water inevitably has to navigate in close proximity of free surface that cannot be considered as "deep immersion". In these regimes the free surface substantially alters the pressure distribution in the hull, therefore all hydrodynamic forces and moments on the body. The pressure field around the body affects the nearby free surface and generates waves which, in consequence change the pressure distribution on the body. With the floating body the wave system is moving that must be "fed" with energy from the forces work on the body. This adds to the wave resistance component and all the other forces and moments on the body.

The motion close the free surface, except occasionally during the some operations, takes place in particular when sailing in the fairway with limited depth. This further complicates the situation. Upon movement of shallow water also result in extra forces and moments due to the influence of the bottom, causing acceleration of the flow around the hull and the spread of the wave system, and accordingly the 
pressure redistribution. The significance of the problem is determined by the specifics of the underwater unit control (inhabited and uninhabited) namely, the reduction in steering performance or loss of control can lead to material or human losses.

The above features are not only important for the water resistance during body motion, hence for the propulsive qualities, but at least for its maneuverability. If in the study of the resistance it is possible to assume that body motion is stationary on a straight trajectory, in the general case of a real maneuvering control we should consider the curvilinear motion with an angle of drift and angular velocity. In this hypothesis of stationarity is now untenable due to the presence of accelerations. Course stability beneath the free surface is subject to research in [6],[10],[11]. In this case the maneuvering qualities of the object changes as a result of the action of three main factors:

- due considered wave making impact not only on the longitudinal force of resistance, but also on the lateral force and moment, caused by drift and angular velocity of the body;

- as a result of the influence of the free surface on the control means characteristics, which are generally symmetrical lifting surfaces with small aspect ratio;

- frequency of oscillation affects hydrodynamic load on the body and in particular generated by control surfaces forces. In conjunction with the wave effects, this may lead to oscillator modes of movement, and in marginal cases - to the bifurcation nature of the motion control process.

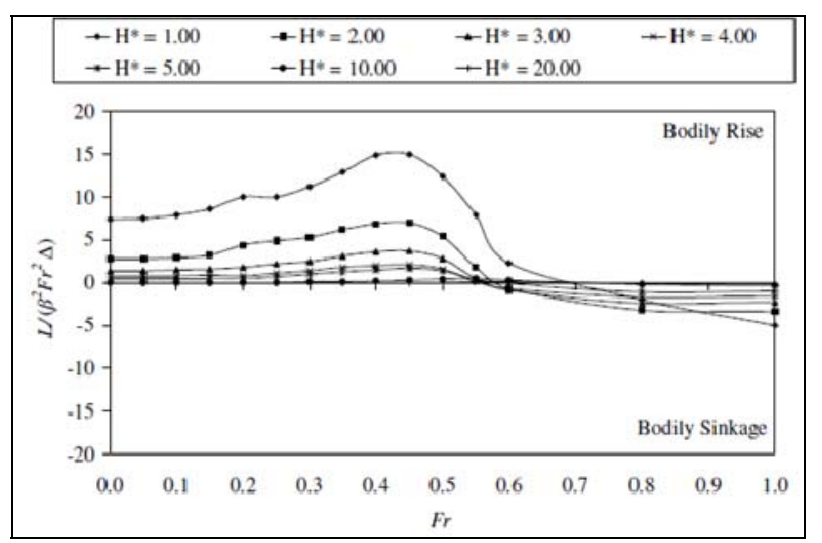

Figure 1. Lift coefficient on the spheroid close to the free surface [12]

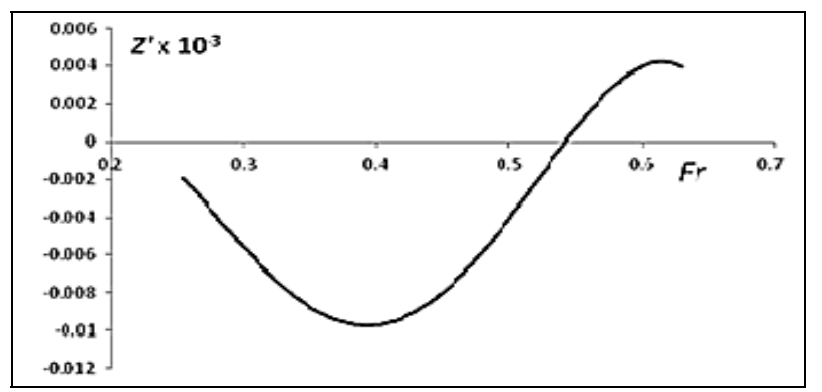

Figure 2. Surface suction force of Suboff geometry with sail only, $\mathrm{H} / \mathrm{D}=1,5[13]$

Regarding the considered factors influence on the hydrodynamics of underwater body close the free surface and the bottom in the literature is available research on single parameters of the phenomenon at a fixed geometry of the body. This necessitates possible global approach to the problem, possibly accounting for the interference effects. As a rule, further underwater bodies have been studied mainly for deep immersion in the basic operating mode, where the resistance and all other forces and moments have a viscous nature, i.e. wave making and induced him wave forces are ignored or regarded as small $[1],[3],[4]$. In recent years, there are studies on the influence of the free surface, experimental, but mainly with numerical simulations [2],[5]. However, they are limited only to some of the effects - the free surface $[2],[5]$ or on the bottom of the flow volume - [7],[8].

\section{OBJECT OF RESEARCH}

Object of present investigations is axisymmetric streamlined elongated reference body, developed for the Defense Advanced Research Projects Agency (DARPA) Suboff Project. Based on the submarine geometry (model 5470), described by Groves et al. [9], the $3 \mathrm{D}$ vehicle model was generated and physical reinforced plastic model have been manufactured Figure 3.

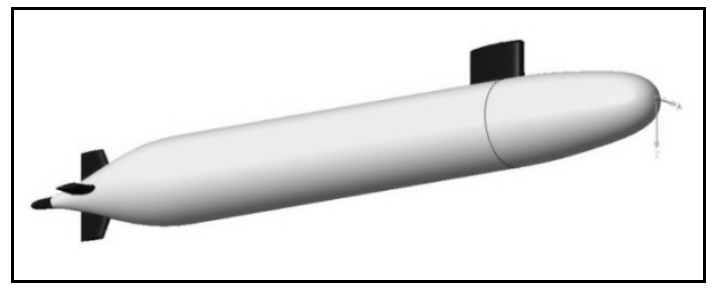

Figure 3. BSHC DARPA Suboff model - fully appended

Principal hull and control surfaces data of the underwater vehicle are given in Table 1.

Table 1. Main particular of the hull and appendages

\begin{tabular}{lcc}
\hline Hull data & Symbol & Values \\
\hline Length overall, m & LOA & 4,356 \\
Diameter moulded, m & $\mathrm{D}$ & 0,508 \\
Rudder lateral area, m2 & $\mathrm{AR}$ & 0,0814 \\
Volume of displacement, m3 & $\mathrm{SWA}$ & 0,718 \\
Wetted surface, m2 & Sh,338 \\
Number propellers & {$[-]$} & 1 \\
Number rudders & {$[-]$} & 4 \\
\hline Control surfaces data & Symbol & Values \\
\hline Rudder area, m2 & $\mathrm{AR}$ & 0,0814 \\
Rudder height, m & $\mathrm{hR}$ & 0,17 \\
Rudder mean chord, m & $\mathrm{bR}$ & 0,184 \\
Aspect ratio & $\lambda \mathrm{R}$ & 0,72 \\
Rudder profile & \multicolumn{2}{c}{ NACA 0020} \\
\hline
\end{tabular}

\section{EXPERIMENTAL SET-UP AND TEST PROGRAM}

The tests were carried out in the deep water towing tank of BSHC which main dimensions are: $200 \mathrm{~m}$ length $\times 16 \mathrm{~m}$ breadth $\times 6.5 \mathrm{~m}$ depth. To meet requirements of submerged body tank testing a project for the modernization of the existing Planar Motion Mechanism was implemented. This creates possibility to carry out captive maneuvering tests to 
determine the course stability derivatives of submerged objects. Specially developed telescopic struts are rigidly connected to the two pair of twocomponent load cells with strain gages for varying the depth of immersion of the underwater body - Figure 4.

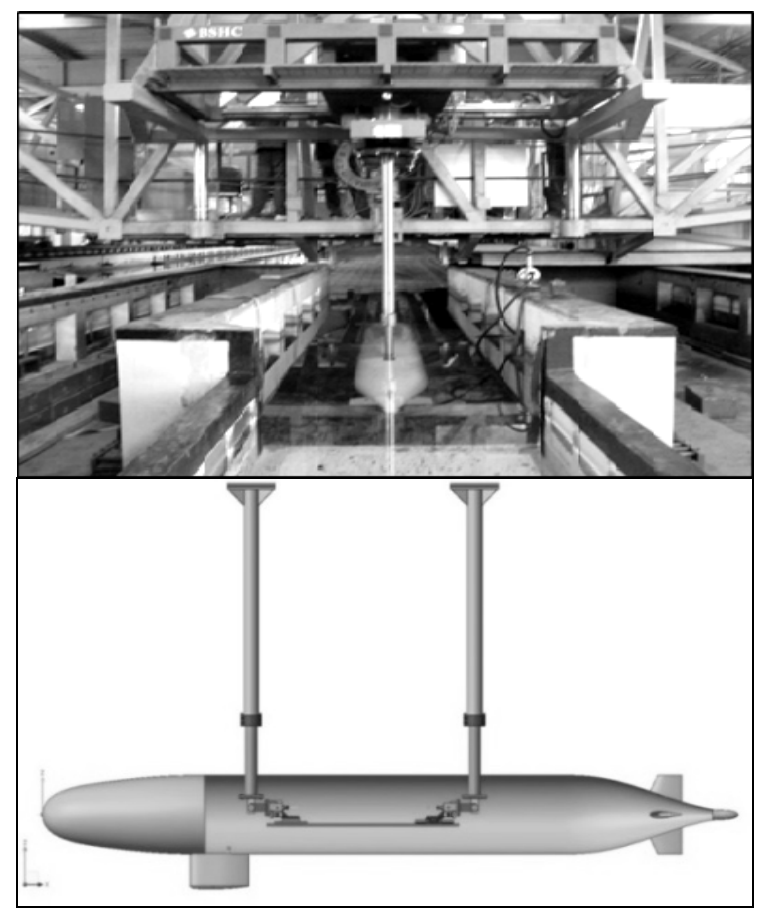

Figure 4. Model installed on the PMM via struts \& load cells

The set-up of DARPA Suboff model experimental maneuvering investigations allows to vary the depth of body immersion and by towing carriage - the linear speed of motion. Due consideration only to the idealized case - course stability in the horizontal plane, assuming the dominant role of the hull forces, without considering the wave resistance and wave pattern, the suboff model is inverted. It is avoids the influence of the struts - the set-up of sail between two struts.

In view of previous studies on the influence of the free surface on the submarine behavior, are selected depths ranging from deep immersion, where there is no such effect, to the critical relative submersion depth, corresponding to the critical Froude number $\left(F n_{h} \approx 1\right)$ related to the influence of shallow water.

$F n_{h}=v / \sqrt{g \cdot H}$

where $\mathrm{V}$ - carriage speed $[\mathrm{m} / \mathrm{s}] ; \mathrm{g}$ - gravitational acceleration $\left[\mathrm{m} / \mathrm{s}^{2}\right] ; \mathrm{H}$ - submergence depth of centreline axis to the free-surface $[\mathrm{m}]$;

Thus BSHC experimental investigations were performed at three depth immersion of the vehicle model, described by data and picture incorporated in the Table 2. The model vertical distance from water free surface was mesured from the model centerline.

The scope of work consists of captive model experiments using scale generic submerged model to quantify the axial resistance (drag force), lateral force (lift) for a submarine operating close to the free surface. Measurements are conducted at a constant forward speed, range submergence depths, and fully appended hull configurations. The tank test matrix has been implemented in two basic modes of model towing: static and dynamic - Table 3.

Table 2 Test depths related to the model center line

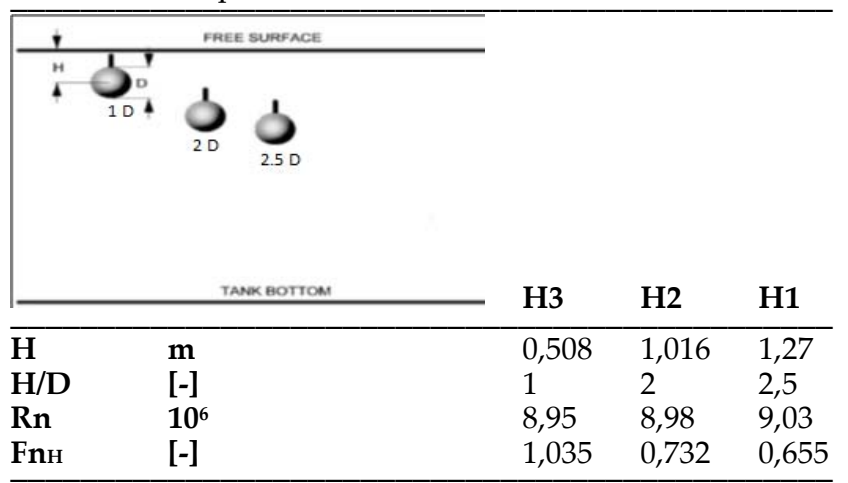

Table 3. Course stability model tests program

\begin{tabular}{|c|c|c|c|}
\hline \multicolumn{4}{|c|}{ STATIC TESTS } \\
\hline Test Mode & $\begin{array}{l}\text { Speed } \\
\mathrm{U}[\mathrm{m} / \mathrm{s}]\end{array}$ & Immersion & $\begin{array}{c}\text { Drift angle } \\
\text { [deg] }\end{array}$ \\
\hline Static drift & 2,31 & $\mathrm{H} 1, \mathrm{H} 2, \mathrm{H} 3$ & $-4,-2,0 ; 2 ; 4$ \\
\hline \multicolumn{4}{|c|}{ DYNAMIC TESTS } \\
\hline Test Mode & $\begin{array}{l}\text { Speed } \\
\mathrm{U}[\mathrm{m} / \mathrm{s}]\end{array}$ & Immersion & $\begin{array}{l}\text { Yaw rate } \\
\mathrm{r}^{\prime} \quad[-]\end{array}$ \\
\hline Pure yaw & 2,31 & $\mathrm{H} 1, \mathrm{H} 2, \mathrm{H} 3$ & $\begin{array}{l}0,04 \\
0,12\end{array}$ \\
\hline
\end{tabular}

\section{COURSE STABILITY ANALYSIS}

An analysis of the stability characteristics of the submarine in horizontal plane was realized making use of dynamic stability indices based on the linearized equations of motion and on hydrodynamic derivatives, obtained by a fore mentioned PMM tests.

According to [12],[13] the underwater vehicle can be considered inherently directionally stable if after a disturbance from steady-state straight motion it resumes its steady motion on another straight direction with controls fixed in zero or neutral position. Below an analysis of DARPA Suboff course stability as a function of body immersion is performed. This is taken into account for two aspects of vehicle course stability, namely:

- inherent to body geometry course stability;

- vehicle response to impulse external actions.

Linear "sway-yaw" model of maneuvering motion in horizontal plane has been considered. The required linear hydrodynamic derivatives were obtained by processing the captive PMM tank tests data.

\subsection{Course stability in horizontal plane}

Obtained by model PMM tests DARPA Suboff at different submergence ratio $H / D$ hydrodynamic derivatives of linearized sway and yaw equations of motion are given in Table 4. Data from other three sources are included, also. 
Table 4 . Non-dimensional stability derivatives in horizontal plane ${ }^{*} 10^{3}$

\begin{tabular}{lllllll}
\hline & $\mathbf{H} / \mathbf{D}$ & $\mathbf{N v}^{\prime}$ & $\mathbf{Y r}^{\prime}$ & $\mathbf{Y v}^{\prime}$ & $\mathbf{N r}^{\prime}$ & $\mathbf{G h}$ \\
& {$[-]$} & {$[-]$} & {$[-]$} & {$[-]$} & {$[-]$} & {$[-]$} \\
\hline DTMB [13] & $\mathbf{6}$ & -13.65 & -12.85 & -27.83 & -4.44 & -0.419 \\
Autosub [17] & $\mathbf{3}$ & -0.45 & 12.64 & -28.45 & -5.35 & 1.037 \\
NPS [16] & n.a. & -7.4 & -43.2 & -100 & -16 & 0.800 \\
\hline \multirow{3}{*}{ BSHC } & $\mathbf{2 . 5}$ & 1.5 & 10.8 & -44.2 & 4.0 & 0.845 \\
& $\mathbf{2}$ & 1.6 & 7.2 & -45.7 & 4.2 & 0.774 \\
& $\mathbf{1 . 5}$ & 2.5 & 5.7 & -59.5 & 4.2 & 0.631 \\
\hline
\end{tabular}

DARPA Suboff inherent course stability has been estimated by means of well-known criterion $G h$ [12],[13]:

$$
G_{h}=1-N_{v}\left(Y_{r}-m\right) /\left[Y_{v}\left(N_{r}-x_{g} m\right)\right]
$$

where: $N_{v}, N_{r}, Y_{v}, Y_{r}$ - first order derivatives of side force and yaw moment in respect to sway velocity $v$ and yaw rate $r$.

Calculated values of criterion $G_{h}$ for the realized test cases, including deeply submerged [13] are given by Figure 5 .

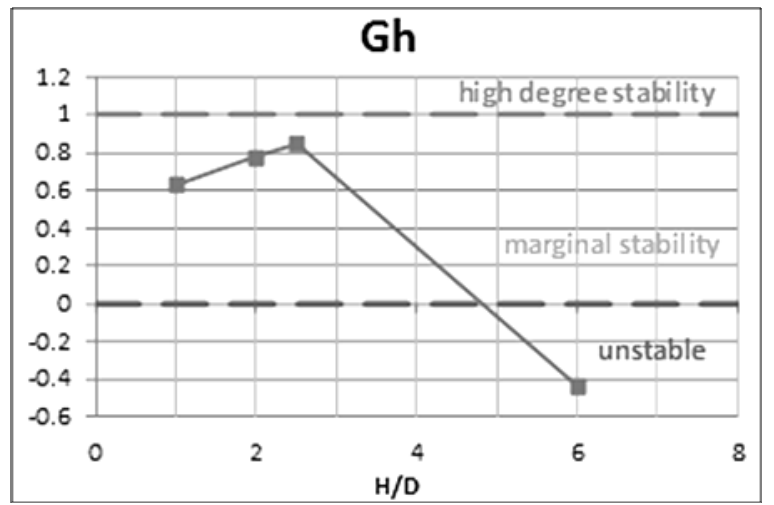

Figure 5. Effect of non-dimensional immersion H/D on Gh

We can conclude that this underwater vehicle is directionally stable close to the free water surface and unstable in deep water conditions. It should be pointed that this submarine feature is inherent to the given body geometry: hull \& sail \& stern controls. Obviously, the operational course stability in both planes will depend on the effectiveness of control surfaces. By the way, to obtain quicker submarine response to control actions in submarine design is observed a tendency to keep small course instability in deep water conditions.

\subsection{Impulse response in horizontal plane}

To investigate the behavior of underwater vehicle when impulse change in motion parameters takes place the linear analysis approach [14],[15] to an open loop control system described by the set of two linear differential equations [13] is applied. Let's suppose that at the moment the ship was subject to instant external sway and yaw disturbance: where: $v_{0}$ and $r_{0}$ - values of the initial impulse actions.

The Laplace transformation of the linearized equations of motion and solution of the system with respect to the side and yaw velocities yields:

$V(p)=v_{0}\left[p\left(a_{1} b_{3}-a_{3} b_{1}\right)+\left(a_{1} b_{4}-a_{4} b_{1}\right)\right]$

$+r_{0}\left[a_{3} b_{4}-a_{4} b_{3}\right] / Q(p)$

$R(p)=r_{0}\left[p\left(a_{1} b_{3}-a_{3} b_{1}\right)+\left(a_{2} b_{3}-a_{3} b_{2}\right)\right]$

$+v_{0}\left[a_{2} b_{1}-a_{1} b_{2}\right] / Q(p)$

The expressions (2) \& (3) have a form of rational functions:

$F(p)=\frac{M(p)}{N(p)}$

In above expression the numerator and denominator are polynomials of the parameter $p$ in which the denominator is of power $n$.

Original of similar function can be found using Heaviside expansion theorem:

$$
f(\tau)=\sum_{i=1}^{n}\left[\frac{M(p)\left(p-p_{i}\right)}{N(p)}\right]_{p=p_{i}} e^{p_{i} \tau}
$$

Than by inverse transform we can obtain expressions for the catamaran response by drift velocity and yaw rate changes when instantaneous disturbance has been applied. Separating above characteristics as four combination of response to particular disturbances in sway and yaw, namely:

$\frac{v(\tau)}{v_{0}} ; \frac{v(\tau)}{r_{0}} ; \quad \frac{r(\tau)}{v_{0}} \frac{r(\tau)}{r_{0}}$

Then we have:

$$
\begin{aligned}
& \frac{v(\tau)}{v_{0}}=\frac{1}{p_{1}-p_{2}}\left[\left(a_{1} b_{3}-a_{3} b_{1}\right) \cdot\left(p_{1} e^{-p_{1} \tau}-p_{2} e^{-p_{2} \tau}\right)\right. \\
& \left.+\left(a_{1} b_{4}-a_{4} b_{1}\right)\left(e^{p_{1} \tau}-e^{p_{2} \tau}\right)\right]
\end{aligned}
$$

$\frac{v(\tau)}{r_{0}}=\frac{1}{p_{1}-p_{2}}\left[\left(a_{3} b_{4}-a_{4} b_{3}\right) \cdot\left(e^{-p_{1} \tau}-e^{-p_{2} \tau}\right)\right.$

$\frac{r(\tau)}{v_{0}}=\frac{1}{p_{1}-p_{2}}\left[\left(a_{2} b_{1}-a_{1} b_{2}\right) \cdot\left(e^{-p_{1} \tau}-e^{-p_{2} \tau}\right)\right.$

$$
v(\tau)_{\tau=0}=v_{0} \quad ; \quad r(\tau)_{\tau=0}=r_{0}
$$




$$
\begin{aligned}
& \frac{r(\tau)}{r_{0}}=\frac{1}{p_{1}-p_{2}}\left[\left(a_{1} b_{3}-a_{3} b_{1}\right) \cdot\left(p_{1} e^{-p_{1} \tau}-p_{2} e^{-p_{2} \tau}\right)\right. \\
& \left.+\left(a_{2} b_{3}-a_{3} b_{2}\right)\left(e^{p_{1} \tau}-e^{p_{2} \tau}\right)\right]
\end{aligned}
$$

Time histories of BSHC DARPA Suboff response to small disturbances in sway and yaw velocities, described by solution in time of equations (4) to (7) for 3 different distances from free water are illustrated by Figure 6 and Figure 7.

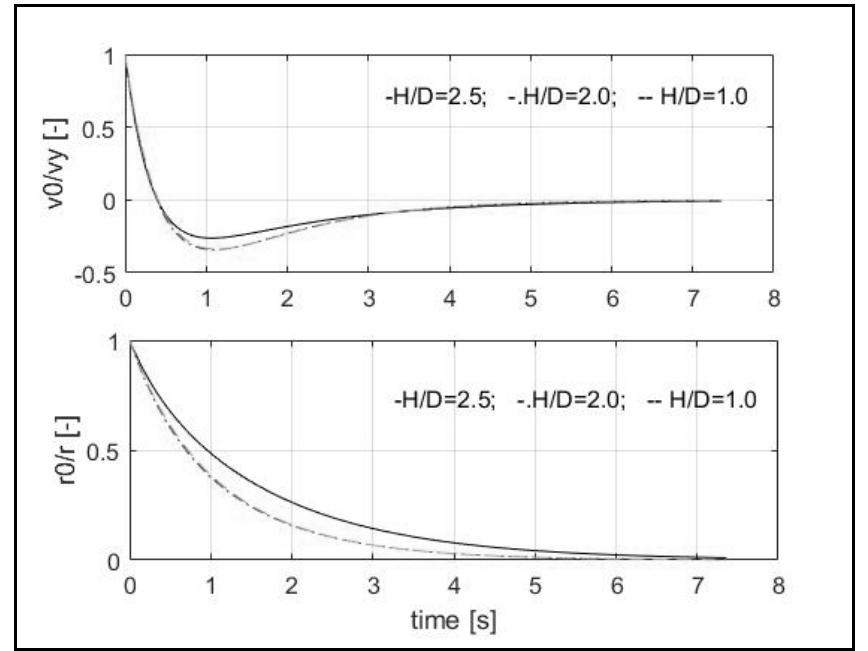

Figure 6. DARPA Suboff response to sway-to-sway and yaw-to-yaw instantaneous disturbance close to the free surface

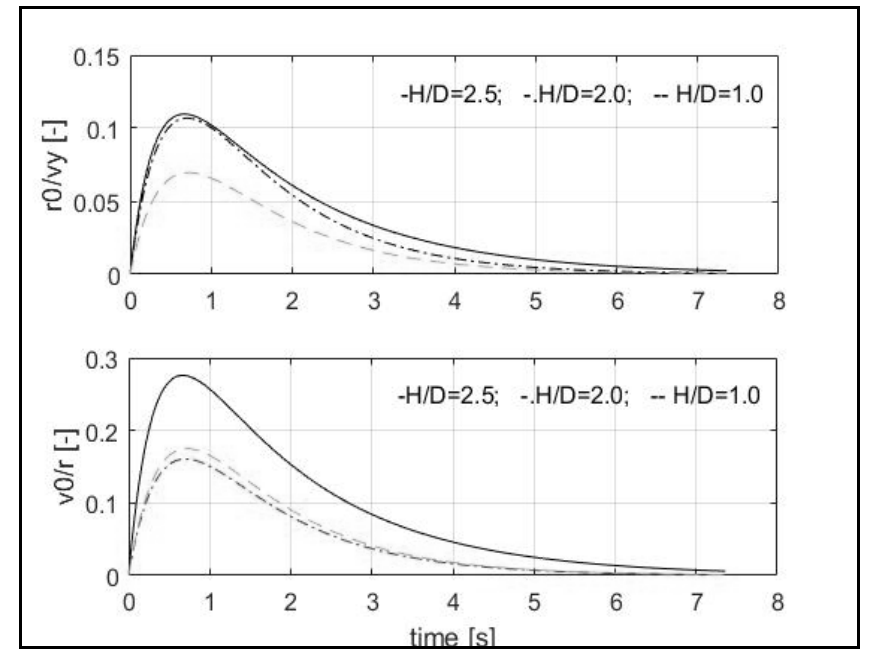

Figure 7. DARPA Suboff response to yaw-to-sway and sway-to-yaw instantaneous disturbance close to the free surface

Based on this it can be concluded that:

- In the case of "yaw-to-yaw" ( $r 0 / r)$ disturbance the motion is critically damped, i.e. the yaw rate decreases uniquely to zero;

- In other cases after impulse disturbances the motion is underdamped, i.e. responses increase/decrease (with sign change) and then go to zero.

\section{CLOSE SURFACE EFFECT INFLUENCE ON THE SUBOFF RUDDER EFFICIENCY}

When considering the vehicle controllability and operational course stability it is of interest to have information about steering forces, generated as a result of control surfaces deflection. In our case we had study the influence of submergence ratio on vertical rudders forces. Thus, hydrodynamic loads on Suboff vertical rudders close to the free surface have been investigated. Calculations for three nondimensional submergence depths at one flow rate were performed, and the data for the two hydrodynamics characteristic parameters of rudder efficiency were derived - drag and lifting force. Ratio of immersion is defined as:

$\mathrm{H}^{*}=\mathrm{H}_{\mathrm{R}} / \mathrm{h}_{\mathrm{R}}$

where: $H_{R}$ - submergence depth of centreline axis to the free-surface, $[\mathrm{m}] ; h_{R}$ - rudder height, $[\mathrm{m}]$

The results of performed numerical study by means of software "ANSYS" of free surface effect on rudder characteristics indicate that with reduction of the depth of immersion the changes of the drag are minimal. This beneficial phenomenon, however, is compensated by the noisy reduction in lifting force the most important factor determining the rudder's effectiveness. In this case, it can be concluded that the performance of the rudder is getting worse as it approaches the free surface. The factor that may be decisive for these results would be the "suction" effect. It would be given rise to by increasing the velocity in the area between the rudder and the free surface, followed by reduced dynamic pressure. Rudder hydrodynamic load (drag and lift) dependency on its immersion for the range deflection angles is shown on Figure 8 and 9 .

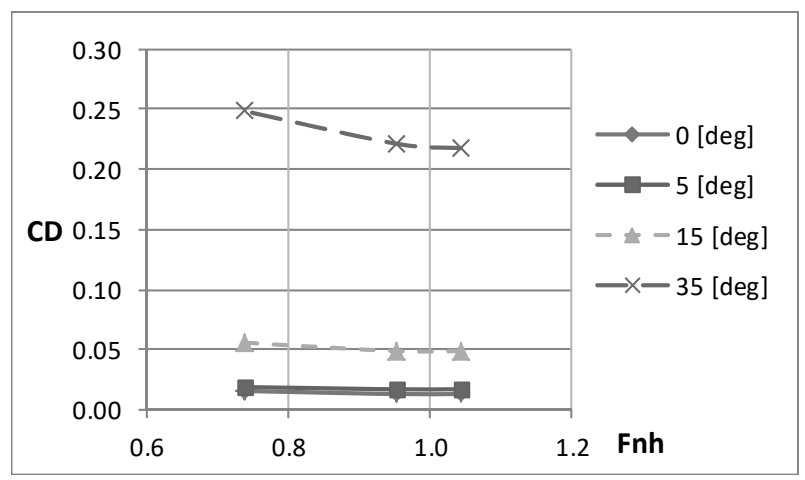

Figure 8. Depth influence on the rudder drag 


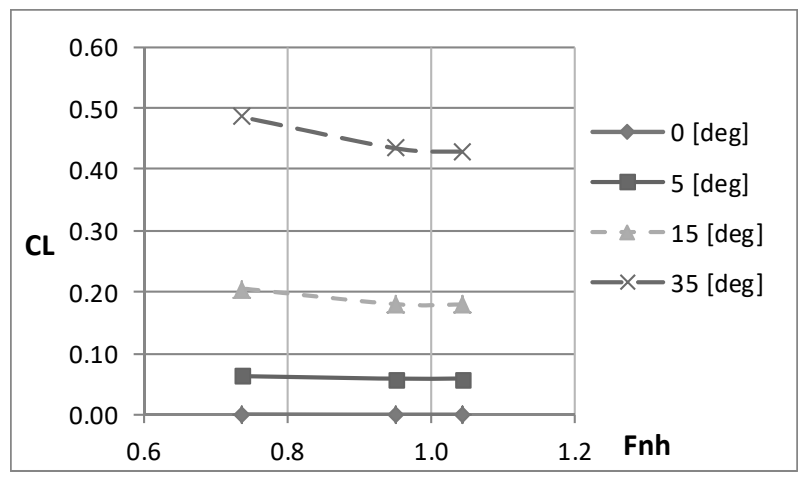

Figure 9. Depth influence on the rudder lift

\section{CONCLUSIONS}

In this study, a DARPA Suboff submarine hydrodynamics for the case of close free water surface operation was investigated. The hydrodynamic coefficients of the maneuvering model have been determined for the three immersions of vehicle by means of specially adopted PMM for underwater bodies tank testing. In such conditions, the course stability in horizontal plane has been estimated and compared with other research data. Similarly, the submarine response to impulse disturbances close to the free surface was calculated. Special attention has been paid to the effectiveness of submarine control surfaces in shallowly immersed conditions.

In the frame of the recent project the research will continue with development of 6DOF maneuvers simulation model for above discussed conditions, which process shall be supported by additional tank tests and numerical studies.

\section{ACKNOWLEDGEMENTS}

This work is performed under Project DM07/6 “ Hydrodynamics of Underwater Body Close to the Free Surface” , funded by Bulgarian National Science Fund at "Competition for financial support for projects of junior researchers - 2016".

\section{REFERENCES}

[1] Joubert, P.N, 2004. "Some Aspects of Submarine Design", Part 1. Hydrodynamics, DSTO Platforms Sciences Laboratory

[2] Jagadeesh, P. \& Murali, 2010. "RANS Predictions of Free Surface Effects on Axisymmetric Underwater Body", Engineering Applications of Computational Fluid Mechanics, Vol. 4, No. 2, pp. 301-313
[3] Han-Lieh Liu \& Huang, T. T., 1998. "Summary of DARPA Suboff Experimental Program Data Experimental Program Data", Naval Surface Warfare Center, CRDKNSWC/HD-1298-1998

[4] Huang, T. T. \& Han-Lieh Liu \& Groves, N. C., December 1989. "Experiments of the darpa suboff program", DTRC/SHD-1298-02

[5] Zhu Q.\& Liu Y.\& Yue D., "Numerical Investigation of Free-Surface Signatures Generated by Submerged Object", http://www.slc.ca.gov/About/Prevention_First/2006/MO TEMS-Numerical-Paper.pdf

[6] Sout S.\& Ananthakrishnan P. , 2011. “Hydrodynamic and Dynamic Analysis to Determine the Directional Stability of an Underwater Vehicle Near a Free Surface", Journal of Applied Ocean Research, vol. 33, pp. 158-167

[7] Phillips A. \& Furlong, M. \&Turnock., S., 2010. “The Use of Computational Fluid Dynamics to Determine the Dynamic Stability of an Autonomous Underwater Vehicle", Proceedings of the Institution of Mechanical Engineers, Part M: Journal of Engineering for the Maritime Environment | Volume:224, (4)

[8] Renilson, M. \& Ranmuthugala, D. \& Dawson, E., Anderson, B., 2011. "Hydrodynamic design implications for a submarine operating near the surface", Warship 2011: Naval Submarines and UUVs, Bath, UK, 29 - 30 June, ISBN No: 1-905040-86-5

[9] Groves N. C.\& Huang, T. T.\&Chang, M. S., March 1989. "Geometric characteristics of darpa suboff models", DTRC/SHD-1298-01

[10] WIGLEY, W.C.S., 1953. "Water Forces on Submerged Bodies in Motion. Transactions", Institute of Naval Architects, 95, pp. 268-279

[11] Griffin, M.J., 2002. "Numerical predictions of manoeuvring characteristics of submarines operating near the free surface.", Ph.D. Thesis in Ocean Engineering at the Massachusetts Institute of Technology

[12] Spencer, B.J., 1968. Stability and Control of Submarines, Journal of the Royal Naval Scientific Service, vol. 23, No.3, UK

[13] Roddy, R.F., 1990. Investigation of the Stability and Control Characteristics of Several Configurations of the Darpa Suboff Model (DTRC Model 5470) from Captive Model Experiments, DTMB Techn. Report DTRC/SHD1298-08, Bethesda, USA

[14] Sobolev, G.V., 1976, Maneuverability of Ships and Ship Control Automation, Sudostroenie, Leningrad, (in Russian).

[15] Milanov E.M. et all, 2010. Numerical and Experimental Prediction of the Inherent Course Stability of High Speed Catamaran in Deep and Shallow Water, Proceedings of 28th Symposium on Naval Hydrodynamics, Pasadena, USA

[16] Barisic, M., 2011. Formation Guidance of AUVs Using Decentralized Control Functions, https://bib.irb.hr/datoteka/530921.Formation_Guidance_ of_AUVs_Using_Decentralized_Control_Functions.pdf

[17] Phillips, A.B. 2010. Simulation of Self Propelled Autonomous Underwater Vehicle, PhD thesis, University of Southampton, UK 